\title{
Visualization of Trajectories of Electron Beams Emitted by an Ion Source with Closed Electron Drift
}

\author{
Ivan V. Bordenjuk, Oleg A. Panchenko, Sergei V. Sologub, and Ian.G. Brown, Fellow, IEEE
}

\begin{abstract}
Trajectories of electron beams emitted by an ion source with an anode layer and Hall electron closed drift orbits were visualized using light emission from a working gas excited by electrons. Gas discharge of magnetron type, arising in the beam drift region under the influence of an electric field of a target bias potential, was visualized.
\end{abstract}

Bias potential in ion-beam etching systems strongly influences the effectiveness of surface cleaning. Ion beams impinging on a target surface can charge the surface positively and cause ion beam deceleration. Ion beam charge neutralization is usually achieved by means of additional devices injecting electrons or negative ions into the positively charged beam [1] or by providing of a longitudinal (with respect to the ion beam direction) electric field, produced by additional source, that leads to the development of non-selfmaintained discharge generating extra-electrons [2]. The magnetic field "sagging" out of the thruster emission slit do not effect on heavy ions that easily reach a target along ballistic trajectories. On the contrary, this field is strong for electrons, and their motion in the drift space is diffusive, and , consequently, it depends on the magnetic lines configuration. The aim of the work described here was to determine trajectories of electrons, emitted from the Hall thruster source with anode layer and moving to a target as well as localization of additional discharge of magnetron type arising in the drift space under the influence of the bias potential electric field.

The basic processes in the Haal thrusters were investigated for a long time [3,4]. Crossed ExB fields provoking electron drift across the electric and magnetic field directions. Suppressed transverse mobility of electrons allows keeping strong transverse electric field. At the same time ions in the plasma are accelerated to the emission slit in the cathode, and their main part easily leave the acceleration region Trajectories of electrons are more complex, that considerably reduces electron emission current density relatively to the ion one. Subsequent electron motion is dependent on the topological

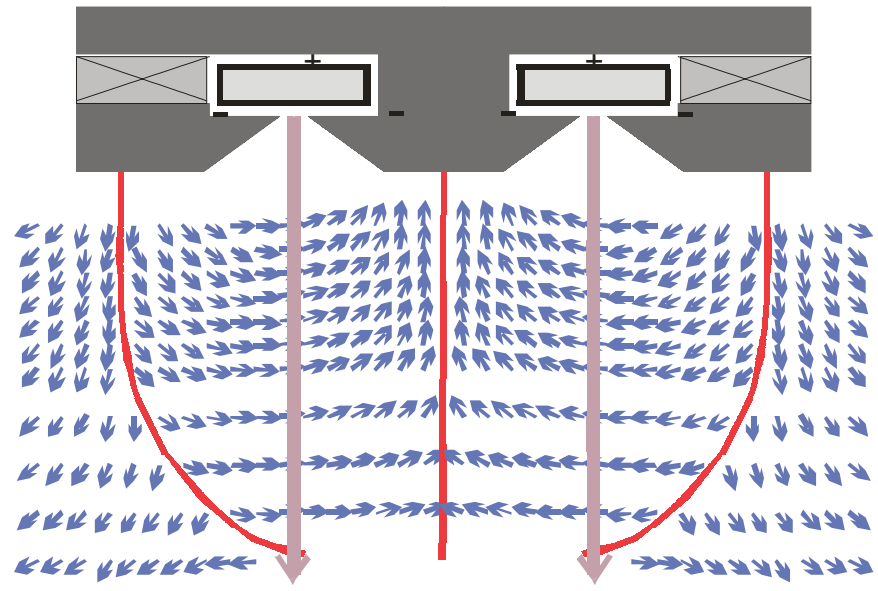

Fig. 1. Magnetic field direction (small arrows) in the ion source drift region is shown in the plane perpendicular to the emission slot plane. The separatrices are shown as red lines, and the ion beams as rose,.

features of the magnetic field within the of beam drift.

The Hall thruster applied in experiments possesses the following characteristics: length of the racetrack emission slit $-250 \mathrm{~mm}$ in the straightforward part and racetrack bending radius - $40 \mathrm{~mm}$, emission slit width - discharge gap $2 \mathrm{~mm}$. Magnet pole pieces were made of soft iron. The watercooled anode with the $23 \times 10 \mathrm{~mm}^{2}$ rectangular cross-section was made of a stainless steel. A thin stainless steel plate $\left(400 \times 150 \mathrm{~mm}^{2}\right)$ was used as a target. Working gas (argon) pressure was within the range from $1 \times 10^{-3}$ Torr to $3 \times 10^{-4}$ ,Torr the base pressure in the vacuum chamber was about $5 \times 10^{-6}$ Torr. The power supply voltage was up to $2.5 \mathrm{kV}$, discharge current - up to $0.3 \mathrm{~A}$. The magnetic field distribution within the drift region is shown in Fig. 1. Arrows indicate the magnetic field direction at that location (and not magnitude). The axial variation in the strength of the radial magnetic field has a bell-shaped distribution and correspondents to the magnetron trap configuration. In the center of the slit magnetic induction was $2 \mathrm{kG}$. Within the 200 .

I.V. Bordenjuk, O.A. Panchenko, and S.V. Sologub are with the Institute of Physics, National Academy of Sciences of Ukraine, 46 Nauki Ave., Kiev, Ukraine 03028.

I.G. Brown is with the Lawrence Berkeley National Laboratory, Berkeley CA 94720.

Work was supported by the STCU (Science \& Technology Center in Ukraine), Project \#118J.

Publisher Identifier S XXXX-XXXXXXX-X 


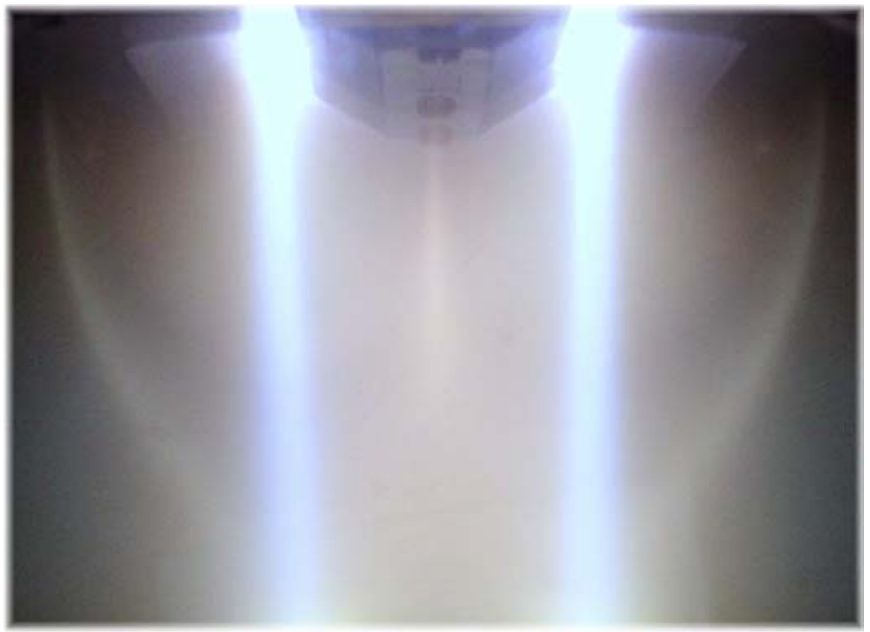

Fig. 2. Photographs of light emission in the drift space for the case of grounded target. The bias potential is about zero.

given field configuration three segments of separatrices were seen (Fig. 1).

Photographs of the lighting discharge region are shown in Fig. 2 and Fig. 3. They were done along the racetrack emission slit at a small angle to its plane. The electron and ion beams trajectories were visualized using light emission from the working gas exited by charge particles. Photograph in Fig 2 was done provided that the ion collector has galvanic connection with the grounded vacuum chamber. Consequently, there is not an electric field induced by the bias potential in the drift space. Thus the light emission induced by ion beams is seeing in the photos as two bright vertical strips. Comparison of Fig 1 and Fig 2 allow us to conclude that the spatial location of the three somewhat less bright emission strips as well as electron beams coincides with the location of the separatrices. Diffuse light emission concentrated between the curved "electron" strips correspondence to the magnetic field configuration in the trap and indicate that the electron motion is mostly normal to the magnetic field lines. The weak light emission out of the lighted region is evidence that the separatrices play role electron collectors and that could attract to the collectors at any point of the beam trajectories.

Photo in Fig. 3 was done in another extreme situation - the target is isolated from the vacuum chamber. At that the longitudinal electric field of the isolated target and the trap magnetic field form a system of crossed ExB fields. It occurs in a comparative narrow region of the drift space where the orthogonal with regard to electric field $E$ component $\mathrm{B}$ of the magnetic field is the maximal (most probably it is the region denoted by pink arrows in Fig. 1). It can conclude from comparison of Fig. 1 and Fig. 3 that in the mentioned drift region in fact the plasma lighting is

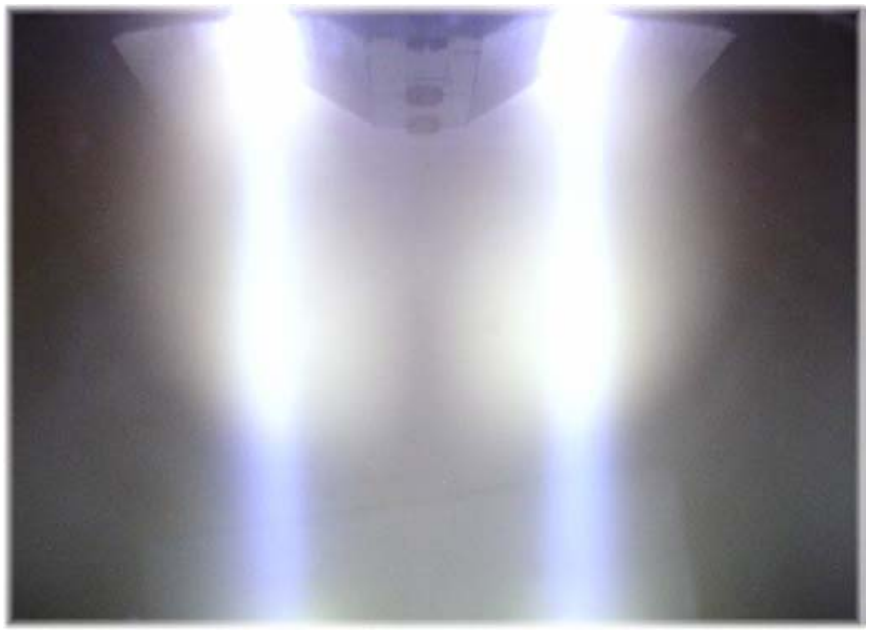

Fig. 3. Photographs of light emission in the drift space for the case of isolated target. The bias potential is about $240 \mathrm{~V}$.

visible. It can be considered as a development of the magnetron type discharge. Because of the longitudinal electrical field of the bias potential and the electrical field in the discharge region are of opposite direction, one can conclude that directions of the Hall drift in these discharges are opposite. The discharge are a source of additional electrons that partially neutralize the target positive charge. Paradoxical is the fact that this additional electron source is not able to provide the total target neutralization because an origin of this discharge is caused by bias potential.

As a result, the magnetic field separatrices play the role of electrons collectors moving from gas discharge to the target. Electrons could attract joint to the electron beams at any point in the beam trajectories. Electric field of the target bias potential can result in to the development of the "secondary" gas discharge of the magnetron type in the drift region.

\section{REFERENCES}

[1] M. G. Gabovich, Physics and technology of plasma ion sources, (in Russian), Moscow, USSR: Atomizdat, 1972.

[2] A. Shabalin, M. Kishinevsky and C. Quinn, "Substrate neutralization methods for the closed drift ion sources", Proceedings of the $44^{\text {th }}$ Annual Technical Conference of Society of Vacuum Coaters, Philadelphia, pp. 23 -28, April 2126, 2001,.

[3] V. V. Zhurin, H.R. Kaufman, and R.S. Robinson, "Physics of closed drift thrusters", Plasma sources Sci. Technol, vol. 8 pp. R1-R20, 1999.

[4] M. Keidar, I. Beilis, "Electron transport phenomena in Plasma devices with $E \times B$ drift", IEEE Transaction on Plasma Science, vol. 34, pp 804- 814, NO. June 3, 2006. 